\title{
Individual Differences in Mood Changes
}

\author{
Magdalena Marszał-Wiśniewska ${ }^{1} \cdot$ Magdalena Nowicka $^{1}$
}

Published online: 26 April 2017

(C) The Author(s) 2017. This article is an open access publication

\begin{abstract}
The study explores the influence of individual tendencies toward mood improvement/deterioration and the Big Five personality traits on mood changes. Participants (218 students) completed NEO-FFI and The Mood Regulation Scales. Based on the within-person structure of individual tendencies toward mood improvement/deterioration four mood regulative types were distinguished (increasing, decreasing, hot and cool type). In the experimental stage participants were randomly assigned to one of four group conditions created by experimental factors: (1) induced mood (positive/negative), and (2) the level of cognitive loading (easy/hard condition). Direct (Mood Adjective Check List) and indirect (emotional version of Lexical Decision Task) measurements were used to assess mood changes. The results showed different patterns of mood changes for increasing and decreasing regulative types. Those differences were visible especially in the positive mood regulation. While the decreasing type decreased the induced positive mood, the increasing type was characterized by mood changes manifested in energetic arousal increase and tense reduction. Moreover, high Neuroticism and low Extraversion in the decreasing type contributed to a negative mood increment manifested in reduction of energetic arousal. The same effect was revealed for high Conscientiousness for the increasing type. The results are discussed in the context of psychological status of mood regulation strategies (automatic/controlled) and with reference to previous research in this area.
\end{abstract}

Keywords Mood $\cdot$ Mood changes $\cdot$ Mood regulation strategies $\cdot$ Individual differences in mood changes

Magdalena Nowicka

nowickamagda@op.pl

1 SWPS University of Social Sciences and Humanities, Chodakowska 19/31, 03-977 Warsaw, Poland 


\section{Introduction}

The subjective well-being (SWB) has been an area of intense research over recent decades. Researchers have been especially interested in the cognitive and affective factors that help to explain individual levels of SWB (Arhaud-Day et al. 2005). SWB increases with the frequency of positive affect and decreases with the frequency of negative affect (Tomyn and Cummins 2011). However, some researchers speculate that high SWB is not necessarily related with low level of negative affect. Accepting one's negative emotions (acceptance) and then trying to seek out positive aspects (positive reappraisal) might be an optimal strategy for building high life satisfaction (North et al. 2011). In other words, negative emotions can contribute to SWB especially when accompanied with adaptive affective regulation strategies. From this point of view a healthy and situation-adequate pattern of mood regulation strategies seems to be critical for SWB.

\subsection{Mood}

The basic affective state known as a core affect refers to the feeling tone component of both emotions and mood (Yik et al. 2011). It is a neurophysiological state experienced constantly and-most of the time-consciously, although the intensity of this feeling varies over time. Examples of core affect include bipolar opposites, namely pleasuredispleasure, tension-relaxation, energy-tiredness. Core affect is not sufficient for a mental representation of emotions which are defined as intense feelings (going beyond pleasure/ displeasure) directed at someone or something (Feldman-Barrett et al. 2007). Mood is understood as a prolonged core affect, often relatively mild, with behavior, thoughts, and motivation. Studies have shown higher correlations of core affect with mood scales than with emotions scales (e.g. Yik et al. 2011). This suggests that mood contains a very large component of core affect. Mood determines life satisfaction and an ability to meet environmental requirements (Larsen 2000). Comparing to emotion, mood is longer, more global, more diffuse and usually remote from its cause. Finally, moods particularly influence cognitive functioning whereas emotions are thought to aid in adaptive reactions.

Different models of mood are usually boiled down to two, mutually dependent, bipolar dimensions: the hedonic dimension and the activation dimension (Diener 1999; Russell 2003; Watson and Tellegen 1985). The neurophysiological data concerning the specificity of the hedonic dimension (Panksepp 1993) and the heterogeneity of the activation dimension ( $c f$. Thayer 1989) led to the formulation of the three-dimensional model of mood (Matthews et al. 1990). This model presents three correlated bipolar mood factors. Two of them are associated with subjective arousal, experienced either as positive affect and energy (Energetic Arousal; energy-fatigue) or as negative affect tension (Tense Arousal; tension-relaxation). A third dimension of Hedonic Tone contrasts pleasant, contented mood with sadness and dissatisfaction (pleasure-displeasure). Schimmack and Grob (2000) showed that this model fits the empirical data best, also semantically, i.e., in terms of the subjective descriptors of experienced affective states.

\subsection{Mood Regulation}

Mood regulation as a part of general self-regulation refers to processes directed toward modifying/maintaining the occurrence, duration, and intensity of mood (Larsen 2000). Different models of mood regulation explain why (motivation approach) (Isen 1987) and 
how (processual approach) (Larsen 2000) people regulate their mood. In motivation approach attention is focused especially on hedonic principle as the major motive underlying mood regulation (Isen 1987). In processual approach mood regulation is conceptualized as a series of distinct but interrelated control processes which rely especially on comparing the desired subjective state (set point) to the current mood (Larsen 2000). When a discrepancy occurs, regulatory mechanisms are engaged to reduce it (negative feedback loop). More and more often researchers come to the conclusion that mood regulation provides a unique route to enhanced well-being and that different functional outcomes of mood regulation depend on different aspects of this processes (Haga et al. 2009; Kashdan 2007). In other words, subjective well-being depends on how, why and when individuals engage in specific strategies to modify or alter their subjective experience.

\subsubsection{Automatic Versus Controlled Mood Regulation}

Classifications of mood regulation strategies are based on two main criteria: (1) the direction of these strategies (mood improvement/deterioration), and (2) the level of their controllability (automatic/controlled) (Larsen 2000).

Whereas the flexible use of strategies leading to mood improvement is associated with enhanced life satisfaction and positive affect, mood deterioration strategies, conversely, contribute to many negative outcomes (e.g. psychopathology) (Kashdan 2007).

Controlled strategies require conscious effort during initiation and implementation whereas automatic strategies operate immediately and independently of conscious control, taking advantage especially of automatic cognitive mechanisms (Larsen 2000). Automatic strategies are perceived as relatively maladaptive, playing an important role in different forms of psychopathology (Mauss et al. 2007). This conclusion is somewhat surprising for two reasons. Firstly, automatic mood regulation is effortless-so probably it is more flexible. Secondly, it seems that many people manage their moods automatically without the above-mentioned negative effect. Furthermore, some unique data suggest that automatic mood regulation may promote resilience, recovery from stress and high subjective well-being (e.g. Coifman et al. 2007). According to dual process-theory automatic and controlled mood regulation strategies are not mutually exclusive categories, but rather have porous boundaries and numerous interconnections (Mauss et al. 2007). A complete understanding of mood regulation processes thus requires a detailed investigation of both automatic and controlled forms of this process, including their possible interactions and contradictory effects. As some authors suggest, their combined as well as dissected effects might be grasped during the procedure of considering the analysis of mood changes in different cognitive loading conditions (Gyurak et al. 2011).

An adequate mood changes measurement is another important methodological challenge when studying mood regulation (Lischetzke et al. 2011). To date mood changes have usually been assessed by direct, self-report questionnaires measuring only momentary mood state and relying on the ability of respondents to report on the construct of interest (Larsen 2000). Meanwhile, mood regulation processes, especially automatic ones, may produce changes which are not always easily accessible for self-insight. Thus, a more precise analysis of automatic and controlled mood changes would be possible, using simultaneously both direct and indirect mood measures (Lischetzke et al. 2011). Indirect measures capture information processing by measuring continuously performance that is relevant to the targeted characteristic and, hence, do not require self-insight. Moreover, they allow to analyze not only momentary states but also the whole course of their changes. 
The research presented here expands the existing data by proposing an experimental design aimed at analyzing both automatic and controlled mood regulation and providing direct as well as indirect measures of mood changes. We measured positive and negative mood changes by assessing the intensity of three mood dimensions twice during the experiment by means of a self-report questionnaire. To provide an alternative to the selfreport we proposed an emotional variant of lexical decision task (LDT) in which participants are asked to categorize verbal stimuli as word or non-word (Niedenthal and Setterlund 1994). The literature regarding affective influences in the LDT showed that moods would facilitate lexical decisions about words specifically related to the their valence (e.g. Halberstadt et al. 1995). According to this foundation sad-induced individuals would respond faster to sad words than to happy words whereas happy-induced individuals would respond faster to happy than to sad words. During this task we decided to manipulate the level of cognitive loading arguing that hard condition (with additional cognitive task) might lead to a greater likelihood of engaging in automatic mood regulation whereas easy condition (without additional task) might promote automatic as well as controlled mood regulation. In developing this procedure we reasoned that mood changes caused by mood regulation processes would be associated with changes in: (1) subjective intensity of different mood dimensions assessed before and after LDT using direct mood measurement (self-report questionnaire), as well as, in (2) time reactions for affective stimuli in LDT (indirect, continuous measure).

\subsubsection{Individual Differences in Mood Regulation}

Individuals differ from each other in their abilities or propensities for engaging in mood regulation (Larsen 2000). Individual differences in mood regulation are related with (1) direction of preferred practices (positive/negative mood increase/decrease) and their psychological status (automatic/controlled, cognitive/behavioral), (2) the frequency of real use of specific strategies, and (3) the individual effectiveness of used practices. In this article we focus on individual tendencies toward mood improvement or deterioration (Larsen 2000; Wojciszke 2003). High tendency toward mood improvement leads to the more frequent use of strategies leading to positive mood improvement/negative mood deterioration, whereas high tendency toward mood deterioration leads to the more frequent use of positive mood deterioration/negative mood improvement.

There is still a shortage of empirical data on stable individual differences in mood regulation (Larsen 2000). So far researchers have explored how often people with different mood regulation tendencies use cognitive strategies leading to negative mood repair and positive mood deterioration. For example, Rusting and De Hart (2000) found that following a negative mood induction, those high in individual tendency to negative mood regulation expectancies experienced more positive memories and recalled more positive words. People with high mood deterioration tendency use positive mood deterioration and negative mood improvement strategies more often (Joormann 2006). Strategies leading to positive mood increase/maintenance are less frequently analyzed empirically (Tugade and Fredrickson 2004). Although these findings begin to stretch the existing conceptualizations of mood regulation to include positive mood improvement/maintenance processes, we still don't know exactly why and how people do it. What is probably more important, there is a lack of research providing experimental evidence supporting the impact of different patterns of mood regulation tendencies on real mood changes (Larsen 2000). Meanwhile, such data could serve as an answer to the following ambiguities. Firstly, we still do not know whether different patterns of mood regulation tendencies produce general changes in mood 
or induce effects that are selective to some dimensions. Previous studies revealed that specific changes of different mood dimensions are modified by different temperamental and motivational traits (Matthews et al. 1990; Zajenkowski et al. 2012). Secondly, the existing evidence supports a model implying that individuals with low mood improvement tendencies use more rarely negative mood repair/positive mood improvement strategies whereas people characterized by low mood deterioration tendencies do not engage in positive mood decrease/negative mood improvement strategies ( $c f$. Rusting and De Hart 2000). What about people with the concomitance of low levels of these traits? Does it means that their moods are more stable? Or maybe the root of their mood changes is in more spontaneous, automatic forms of mood regulation? Thirdly, studies suggest that high tendency toward mood deterioration is definitely maladaptive, whereas high tendency toward mood improvement leads to positive psychological outcomes (Haga et al. 2009). Meanwhile, the combination of these two traits may be quite hazardous. On the one hand, they may activate contradictory strategies leading to negative affective consequences and psychopathology. On the other, their synergistic working can result in more flexible mood regulation and higher SWB.

The present research attempts to contribute to previous data by exploring the impact of different patterns of mood regulation tendencies on automatic and controlled mood changes. We decided to begin our investigation by determining the within-person structure of individual tendencies toward mood improvement/deterioration through a cluster analysis. We assumed that this method would allow an individual to fall into naturally occurring groups rather than requiring the artificial creation of all possible combinations of variables (Gohm 2003). The process of forming relatively homogenous groups makes data more manageable and facilitates communications. Moreover, the cluster analysis gives the opportunity to make a greater contribution to the area of SWB through the identification of specific groups that might best benefit from different types of interventions concerning mood management.

\subsection{Personality and Mood Variables}

Although many variables (i.e., subjective well-being, emotional intelligence etc.) may be meaningful for examining individual differences in affective functioning, researchers suggest that the relation between personality traits and mood seems to be the most important (Augustine and Larsen 2015; Gross 1998). Mood is essential for our understanding of numerous personality and individual differences variables. Affective states can be inputs, components, or outputs of the personality system. Some results suggest that personality predicts more than one-third of the variance along the dimension of Pleasantness-Unpleasantness and less than a third of the variance along the dimension of Activation-Deactivation (Augustine and Larsen 2015; Yik and Russell 2001).

A significant portion of the research to date has focused on the relation between basic mood components and Big Five personality traits. Extraversion and Neuroticism are the two dimensions most predictive of momentary affect (Diener 1999; Rusting and Larsen 1995; Watson and Clark 1997). Extraversion is linked strongly and positively to levels of positive affect and less negative affect variance, whereas neuroticism-to level of negative affect and less positive affect variance. However, data concerning the tripartite model of affect suggest that the relation between major personality dimensions and mood is not as clear as it appears to be at first glance. For instance, Matthews et al. (1990, 2003) reported a correlation only of around .25 between neuroticism and tense arousal, and .13 between extraversion and positive affect. This group of results relates to experimental studies where 
measuring mood forms a part of a broader procedure and usually occurs prior to performance. It may indicate that the relationships between major personality traits and mood dimensions are not stable and vary across situations (Zajenkowski et al. 2012). In other words, the demands of a situation (e.g. cognitive demands) may be another factor that influences the relation between personality and mood as well as between personality and the specificity of mood changes. However, studies which brought these interesting data were based only on self-reports instruments of analyzed mood changes (e.g. Matthews et al. 1990) and considered only two measurements of mood (before and after the stimulus presentation).

Although there are probably many possible consequences of Big Five personality traits on affective experience, the current research focuses on the consequences for mood changes processes, including both positive and negative mood. So far, researchers have found that mood deterioration strategies (e.g. rumination) correlate positively with neuroticism and negatively with extraversion, whereas mood improvement strategies (e.g. cognitive engagement) correlate positively with extraversion (Augustine and Larsen 2015; John and Gross 2007). Conscientiousness predicts positively the use of strategies involving behavioral engagement and negatively-waiting strategies (Augustine and Larsen 2015). Those high in agreeableness are more likely to engage in affect repair in social settings whereas those high in openness are more likely to use strategies involving active distraction, behavioral engagement and rumination (Tobin et al. 2000). Some authors suggest that specific effects of personality traits on mood regulation may also depend (like personality-mood relation) on the specific features of the situation in which regulatory efforts takes place (John and Gross 2007). This area still requires a definite answer. Moreover, we still do not know whether personality traits have an analogical impact on controlled and automatic mood regulation strategies. Some data suggest that personality traits composed structurally of affective experience modify both automatic and controlled mood regulation, whereas these non-affective in structure-only controlled strategies (Gyurak et al. 2011). Such relationships have not been studied more precisely yet.

The current project focuses on the impact of Big Five personality traits on automatic and controlled changes of both positive and negative mood. Moreover, this study expands the existing data by examining the possible interactional effects of Big Five personality traits and different patterns of mood regulation tendencies on real mood changes. As the existing data have shown, relations between these constructs are rather modest in size (Gross and John 2003). It suggests that mood regulation tendencies converge with, but did not duplicate, broader personality dimensions. Thus, they may contribute differently to predict different aspects of well-being and life satisfaction (Haga et al. 2009).

\subsection{The Aims and Hypotheses of the Present Study}

The first important aim of the current study was to analyze the influence of different patterns of individual tendencies toward mood improvement/deterioration on automatic and controlled changes of both positive and negative mood. The second aim was to learn how Big Five personality traits would modify mood changes. We posed three hypotheses.

H1 Mood changes related with high tendency toward mood improvement consist in positive mood increasing and negative mood decreasing.

H2 Mood changes related with high tendency toward mood deterioration rely on positive mood decreasing and negative mood increasing. 
H3 Mood changes for high neurotic individuals consist in positive mood decreasing (after positive mood induction) and negative mood increasing (after negative mood induction), whereas for high extraverts - in positive mood increasing (after positive mood induction) and negative mood decreasing (after negative mood induction).

Moreover, we attempted to contribute to the previous research by formulating the following empirical question:

Q1 How do interactions of individual tendencies toward mood improvement/deterioration and Big Five personality traits modify automatic and controlled mood changes?

\section{Methods}

\subsection{Participants}

A total of 218 undergraduate psychology students of SWPS University participated in the study. The sample consisted of 132 female and 86 male participants with a mean age of 24.3 years $(S D=4.14)$. Participants were required to be fluent in Polish, to have no history of psychiatric disorders, to be right-handed, and to have normal or corrected-tonormal vision. All participants were volunteers and they participated in the research individually and anonymously. They provided a written consent according to the procedures of the Ethics Committee of SWPS University.

\subsection{The Design and Procedure of the Study}

The presented research comprised two stages:

1. A questionnaire based study in which individual tendencies toward mood improvement/deterioration and the Big Five personality traits were measured.

2. An experiment (one week after the 1st stage) used to test changes of positive and negative mood in two levels of cognitive loading.

In the experimental stage (see Fig. 1), participants were randomly assigned to one of four between-groups conditions created by the factorial combination of induced mood (positive/negative) and the level of cognitive loading (low/high cognitive loading condition). Subsequent steps of the experiment were as follows. First, in order to measure their current mood state, participants completed Mood Adjective Check List, MACL (control measurement before mood induction). Secondly, they were trained in lexical decision task, LDT. Training, typically applied in this type of procedure, served to practically acquaint the participants with instruction, fixation point, and the pattern of responding (Niedenthal and Setterlund 1994). By using training we tried to ensure that stimulus identification speed

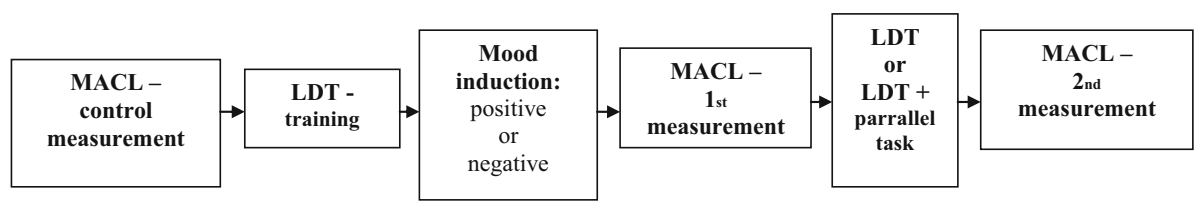

Fig. 1 The scheme of the experimental procedure 
in the main part of experiment was mediated especially by affective information processing and not by disturbances related to technical aspects of procedure.

Following the training, the participants were exposed to mood manipulation. They were reading (for $7 \mathrm{~min}$ ) about 250-word long, sad (excerpt of the testimony of a homeless person) or happy story (an excerpt of a funny family tale) presented on the computer screen while listening to background musical pieces (happy/sad). Next, to assess the effects of this procedure on their mood state the participants rated their current mood by MACL (1st direct mood measurement). It was used firstly, before $L D T$, as a momentary, subjective, requiring self-insight and more precise mood measure. Levels of three mood dimensions assessed by MACL in 1st measurement served as a baseline for assessing mood regulation processes taking place during the next part of the experiment. Moreover, this measurement possibly increased the situational clarity of feelings, which is indispensable in order to activate different mood regulation processes (Larsen 2000; Lischetzke et al. 2011). After the 1st direct mood measurement by MACL participants completed an emotional version of $L D T$ which was used as an indirect continuous measure of mood changes. In hard condition participants were also asked to perform a parallel task (automatic mood regulation condition). $L D T$ (in easy as well as in hard condition) took approximately 7-9 min to complete. The last direct mood assessment by MACL took place after the lexical decision test (2nd measurement). This measurement served as an endpoint for mood regulation processes.

Although the afore-described procedure is quite complicated, it allows to grasp both automatic and controlled processes related to different aspects of emotion regulation. Data from previous studies showed that RTs for affective stimulus in LDT reliably predict mood changes at subsequent measurement occasions in easy and hard condition (Nowicka 2009; see also Lischetzke et al. 2011).

\subsection{Measures}

\subsubsection{Mood Regulation Scales}

In order to measure individual tendencies toward mood improvement/deterioration The Mood Regulation Scales (MRS; Wojciszke 2003) were used. The MRS comprises two subscales: Mood Improvement (MIS) and Mood Deterioration (MDS). MIS includes 11 items describing cognitive and behavioral strategies leading to positive mood increase and 4 items related to negative mood decrease (for example; When I feel well, my memory goes back to pleasant moments, I try to find a positive side of a bad situation). MDS is composed of 12 items representing activities leading to positive mood decrease and three items concerning mood increase practices (for example: When I am happy, I start thinking that it is only an illusion, When I am in a bad mood, I stop doing all pleasant things). The respondents are requested to answer the question (using a five point Likert scale from never to always) of how often they use different regulation strategies.

An exploratory factor analysis of the MRS (principal component, Varimax rotation, Kaiser's normalization) conducted on the data from two independent samples (Wojciszke 2003) supported two factors solution (tendency to Mood Improvement and tendency to Mood Deterioration). Scales of MRS are independent (non-significant inter-correlation coefficients ranging from .03 to -.18) and obtained high internal reliability scores in validation studies (Cronbach alphas for MIS and MDS are .84 and .90 respectively; Wojciszke 2003) as well as in presented study (Cronbach alphas for MIS $=.82$ and $M D S=.87)$. 
Convergent and discriminant validity of the MRS questionnaire was examined in previous research (Nowicka 2009; Wojciszke 2003) by analyzing the relationship between two scales of MRS and different measures related to emotion regulation (Emotion Regulation Questionnaire-Gross and John 2003; Cognitive Emotion Regulation Questionnaire-Garnefski et al. 2001), emotional functioning (Trait Meta-Mood Scale-Salovey et al. 1995; The Positive and Negative Affect Schedule-Watson et al. 1999) and personality (NEO-FFI, Costa and McCrae 1992; Life Orientation Test-Revised, Scheier et al. 1994). $M D S$ correlated strongly positively with non-adaptive emotion regulation strategies [e.g. for Suppression $r=.28, p<.001$; for Catastrophizing $r=.57, p<.001$, Negative Affect $(r=.39, p<.01)$ and negatively-with Positive Affect $(r=-.35, p<.01)$. MIS, in contrast, showed high positive correlation with emotional processing $[r=.52$, $p<.001]$, mood repair $[r=.63, p<.001]$, moderate positive correlations-with adaptive emotion regulation strategies [e.g. for Reappraisal-r $=.33, p<.001$; for Refocus on planning $r=.54, p<.001]$, Positive Affect $(r=.37, p<.01)$ and negative moderate correlation-with Negative Affect $(r=-.25, p<.01)$. Correlation coefficients between mood regulation tendencies and personality traits were modest in size, indicating that two scales of $M R S$ converge with, but did not duplicate basic personality dimensions (i.e. Gross and John 2003). MDS correlates positively with Neuroticism $(r=.24, p<.05)$, whereas MIS correlates positively with Extraversion $(r=.23, p<.05)$, Openness to Experience $(r=.18, p<.05)$, Agreeableness $(r=.21, p<05)$, and dispositional Optimism $(r=23$; $p<.05)$. Correlation coefficients between Big Five personality traits and MRS scales obtained in presented study were very similar, ranging from .11 to .23.

Additionally, we analyzed the relation between scales of MRS and NEO-FFI more precisely by employing the principal factor analysis with Varimax rotation for all the items of these two questionnaires. It revealed that a seven-factor solution fits the data best. The seven-factor solution accounted for $59.9 \%$ of the total variance. Visual inspection of the rotated item-factor loadings suggested that the factors represented Neuroticism (15\% of the total variance), Extraversion (11.9\%), Agreeableness (11.3\%), Openness to Experience $(10.7 \%)$, Consciousness, Mood Deterioration (with four high loading items from Neuroticism scale; 5.6\%), Mood Improvement (with three high loading items from Extraversion scale and two high loading items from Openness to Experience scale; 5.4\%). These results may suggest that Big Five dimensions and mood regulation tendencies are interrelated but not strongly overlapping constructs.

\subsubsection{NEO-FFI Questionnaire}

The Big Five personality traits were measured by NEO-FFI questionnaire (Costa and McCrae 1992) in the Polish adaptation by Zawadzki et al. (1995). The internal reliability scores of this questionnaire (Cronbach alphas) in the Polish national sample are satisfactory and achieve values ranging from .64 to .84. Analogical coefficients (ranging from .68 to .80$)$ were obtained in the present study.

\subsubsection{Mood Adjective Check List (MACL)}

In the experimental stage mood was directly measured using the Mood Adjective Check List (MACL) by Matthews et al. (1990) in the Polish adaptation by Gorynska (2005). The respondent is requested to answer the question Does this adjective describe your present mood? on a 4-point scale from definitely not to definitely yes. The check list has three scales corresponding to the three dimensions of mood (Matthews et al. 1990, 2003): hedonic tone 
$(H T)$, tense arousal (TA), and energetic arousal (EA). The Polish adaptation of the MACL was standardized on a national representative sample. An exploratory factor analysis supports three dimensions of mood. The internal reliability scores were satisfactory in the Polish validation study [Cronbach alphas for $H T=.89, T A=.93$, and $E A=.78$ (Gorynska 2005)], as well as, in the present study [Cronbach alphas for $H T=.86, T A=.80$, and $E A=.74]$.

\subsubsection{Mood Induction}

In the mood induction procedure participants were asked to read one of two stories differentiated by their affective valence (positive or negative), while listening to background music pieces (happy or sad). They were reading an excerpt of a funny family tale versus an excerpt of the testimony of a homeless person while listening to background musical pieces: Scott Joplin's Pine Apple Rag and Eric Satie's Gnossienne no 1 for positive and negative mood, respectively. As the preliminary study showed, both stories as well as both music pieces had the affective impact of comparable strength (Nowicka 2009).

In the preliminary analysis [three one-way repeated-measures ANOVAs (induced mood as the factor and a given dimension of mood as dependent repeated variable] we verified the mood induction procedure by comparing the control mood measurement and the 1st mood measurement after mood induction. The participants in whom a positive mood was induced, compared with those in whom a negative mood was induced, had significantly higher mean scores on the scales of $H T\left(F_{(1,216)}=16.82, p<.001, \eta^{2}=.29 ; M=3.28\right.$, $S D=.58$ vs. $M=2.74, S D=.31$, respectively $)$, and of $E A\left(F_{(1,216)}=23.37, p<.001\right.$, $\eta^{2}=.30 ; M=3.33, S D=.62$ vs. $M=2.81, S D=.41$, respectively), and significantly lower mean scores on the scale of $T A\left(F_{(1,216)}=32.02, p<.001 ; \eta^{2}=.33 ; M=2.74\right.$, $S D=.47$ vs. $M=2.96, S D=.59$, respectively).

The efficiency of mood induction was also verified in the preliminary study (Nowicka 2009). We measured the participants' mood just before and just after the mood induction procedure described above (students, $N=130, M_{\text {age }}=26.13, S D=3.56$ ) by means of the MACL questionnaire. We did not use any additional task. A series of three one-way repeated measures ANOVAs (induced mood as the factor and a given dimension of mood as dependent repeated variable) showed significant effects (for $H T: F_{(1,128)}=18.33$, $p<.001, n^{2}=.31$; for $T A: F_{(1,128)}=24.33, p<.001 ; n^{2}=.28$; for $E A: F_{(1,128)}=23.45$; $\left.p<.01 ; n^{2}=.23\right)$. In the second measurement participants in whom a positive mood was induced, compared with those in whom a negative mood was induced, had significantly higher mean scores on the scale of $H T(M=3.18, S D=.63$ vs. $M=2.63, S D=.28$, respectively), and of EA ( $M=3.41, S D=.57$ vs. $M=2.83, S D=.39$, respectively), and significantly lower mean scores one the scale of TA $(M=2.54, S D=.36$ vs. $M=3.13$, $S D=.44$ respectively). We also found that the mood induction procedure modified significantly the intensity of mood dimensions in each group. After the positive mood induction the level of $\mathrm{HT}\left(M_{1}=2.84, \mathrm{SD}=.31\right.$ vs. $\left.M_{2}=3.18, \mathrm{SD}=.23\right)$, and $\mathrm{EA}$ $\left(M_{1}=2.77, S D=.16\right.$ vs. $\left.M_{2}=3.03, S D=.29\right)$ significantly increased in second measurement whereas the level of TA decreased $\left(M_{1}=2.84, \mathrm{SD}=.23\right.$ vs. $\mathrm{M}_{2}=3.13$, $\mathrm{SD}=2.51$ ). After the negative mood induction the pattern of results was reversed (for HT: $M_{1}=2.74, S D=.16$ vs. $M_{2}=2.51, S D=.21$; for $\mathrm{EA}: M_{1}=2.76, S D=.21$ vs. $M_{2}=2.43, S D=.26$; for TA: $M_{1}=2.61, S D=.36$ vs. $M_{2}=3.18, S D=.34$ ). 


\subsubsection{Lexical Decision Task}

In order to measure mood changes indirectly, an emotional version of the lexical decision task (LDT) was used (Halberstadt et al. 1995; Niedenthal and Setterlund 1994).

During the main procedure of LDT on each of 108 trials (in training session-18 trials), participants were presented with strings of letters and were instructed to indicate whether the presented string was a legitimate Polish word or not (easy condition). Additionally, in hard condition participants were instructed to perform a parallel task which consisted in deciding whether the image (arrangement of two straight lines and three stars) presented on the computer screen was identical with the pattern shown when giving the instructions. We also manipulated the level of cognitive loading in the course of training. All decisions had to be done as quickly and as accurately as possible. In the easy condition participants responded by pressing the "P" key with their right index finger for "word" and the "Q" key with their left index finger for "non-word". In the hard condition individuals responded by pressing (with the index finger of their right hand) the "P" key for "word" or the "L" key for "non-word", and simultaneously (with the index finger of their left hand) the "Q" key, if the image was identical with the pattern or the "A" key if the image was different. The letter strings consisted of 27 words [nine positive (e.g., delighted), nine negative (e.g. aggressive), nine neutral (e.g. wooden)] and nine non-words. During the main procedure all stimuli were grouped into nine experimental blocks each consisting of three positive, three negative, three neutral and three non-words (pseudo-homophones). Non-words were pronounceable and closely resembled words, typically differing in two letters. They were matched on concreteness, frequency, imageability, pronounceability, and the number of letters. Additionally, all affective stimuli were judged by 15 psychology students on a scale of -3 (very negative) to +3 (very positive). Positive, negative, and neutral words had mean ratings of $2.56(S D=.29) ;-2.02(S D=.48)$ and $.33(S D=.11)$ respectively. During the training session the stimuli were presented in two blocks, each consisting of 6 neutral stimuli and 3 non-words.

In the easy condition the onset of each trial was marked by a horizontally and vertically centered "plus" sign $(+)$, which served as a fixation point. In the hard condition two points $(+)$ appeared in the middle of the each half of computer screen and were replaced by a string of letters (right side) and the image (left side). After $500 \mathrm{~ms}$ latency, the points was replaced by the stimuli. The stimuli items disappeared after the participant responded or following a latency of $10,000 \mathrm{~ms}$, whichever occurred first, and were followed by an intertrial interval of $200 \mathrm{~ms}$. All stimuli in trials were presented in a randomized fashion, controlled by computer software.

In the preliminary study we verified that the above procedure of $L D T$ with training session (as well as training treated as a separate procedure) not proceeded by mood induction had no impact on subjective mood state measured just before and just after its performance. A series of repeated measures ANOVAs [for the whole procedure: the level of cognitive loading as the factor and a given dimension of mood as dependent repeated variable; for training: a given dimension of mood as dependent repeated variable] did not show significant changes for three basic mood dimensions [LDT with training: for $H T$ : $F_{(1,156)}=8.12, p=.09, \eta^{2}=.091 ; M_{1}=2.91 ; M_{2}=3.01 ;$ for $E A: F_{(1,156)}=10.37$, $p=.11, \eta^{2}=.018, M_{1}=2.89 ; M_{2}=2.83$, and for $T A: F_{(1,156)}=8.0, p=.13$; $\eta^{2}=.014 ; M_{1}=2.54 ; M_{2}=2.63 ;$ for training: for $H T: F_{(1,157)}=12.12, p=.11$, $\eta^{2}=.099 ; \quad M_{1}=3.04 ; \quad M_{2}=3.02 ;$ for $E A: F_{(1,157)}=12.48, \quad p=.24, \quad \eta^{2}=.010$, 
$M_{1}=3.01 ; M_{2}=2.97$, and for $T A: F_{(1,157)}=10.0, p=.18 ; \eta^{2}=.009 ; M_{1}=2.61 ;$ $\left.M_{2}=2.67\right]$.

For each of the stimuli presented in LDT RTs were unobtrusively measured. We excluded from the analysis data RT outliers (any RTs less than $250 \mathrm{~ms}$ or greater than $1.500 \mathrm{~ms}$ ) and incorrect responds to RTs. It resulted in less than $2 \%$ of the original data being removed. Because of the disturbances of distribution RTs for all stimuli were transformed using natural logarithm.

\section{Results}

\subsection{Patterns of Individual Tendencies Toward Mood Improvement/ Deterioration}

In the first step we analyzed the within-person structure of tendencies toward mood improvement/deterioration and we developed the typology of persons.

The MRS scores were first standardized across the whole sample. To group participants according to the profile of their scores on mood regulation scales, K-means cluster analyses on the Euclidean distances between scores was conducted. The four-cluster solution provided the maximum amount of distinctiveness among the participants while keeping within cluster variance to a minimum and the cluster sizes large enough to allow analysis of different experimental conditions. In Fig. 2, for four groups defined in the cluster analysis, the profiles of mood improvement and mood deterioration are given.

Clusters $1(N=52)$ and $2(N=60)$ are mirror images of one another and were labeled decreasing type (individuals scoring above the mean on mood deterioration and below on mood improvement) and increasing type (individuals scoring above the mean on mood improvement and below on mood deterioration) (e.g. Gohm 2003). Participants in cluster 3 $(N=41)$ scored below the mean on two scales and were labeled cool type. Cluster 4 $(N=56)$, named as hot type, consisted of participants who scored above the mean on two

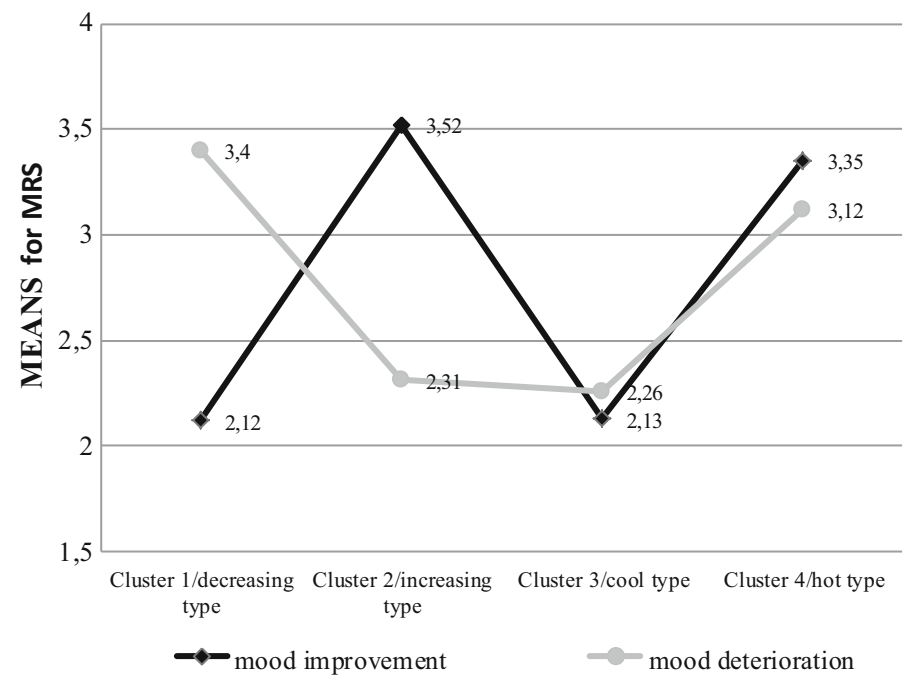

Fig. 2 Results of K-means cluster analyses 
scales. One-way analyses of variance (ANOVAS) were conducted to examine mean differences in two types of mood regulation strategies inside each cluster. In line with our expectations mood deterioration and mood improvement scores differ significantly only in cluster $1\left[F_{(1,51)}=16.14 ; p<.001 ; \eta^{2}=.022\right]$ and $2\left[F_{(1,59)}=5.38 ; p<.05 ; \eta^{2}=.055\right]$ (see Fig. 2). Four regulative types distinguished on the basis of cluster analysis were included in further analyses.

We verified general pattern of results obtained by individuals representing each cluster in NEO-FFI questionnaire. The analyses of variance (MANOVAS) showed that the profile of results differed between clusters $\left[F_{(5,197)}=94.01 ; p<.05 ; \eta^{2}=.047\right]$. Figure 3 displays personality profiles of each cluster as summarized by mean NEO-FFI $T$ scores. Decreasing type was characterized by higher neuroticism and lower extraversion, openness, agreeableness, and conscientiousness scores than hot, cool and increasing type $(p s<.01)$. The pattern of results obtained in NEO-FFI by increasing and hot type was incredibly similar. They scored higher on extraversion than cool and decreasing type ( $p$ s $<.01)$ and lower on neuroticism than decreasing type $(p s<.01)$.

\subsection{Individual Differences and Automatic and Controlled Mood Changes}

To examine the impact of regulative types on mood changes we used the four-way ANOVAs with direct mood measure (level of HT, EA and TA) and with indirect mood measure (time reaction in LDT) as dependent repeated variable, and regulative types, moment of measure, type of mood induction, and the level of cognitive loading as independent variables. Analogical five-ways ANOVAs with additional independent variablethe level of Big Five personality traits were made (separate analysis for each trait of Big Five). A median split was used to separate people with high and low levels of Big Five personality traits.

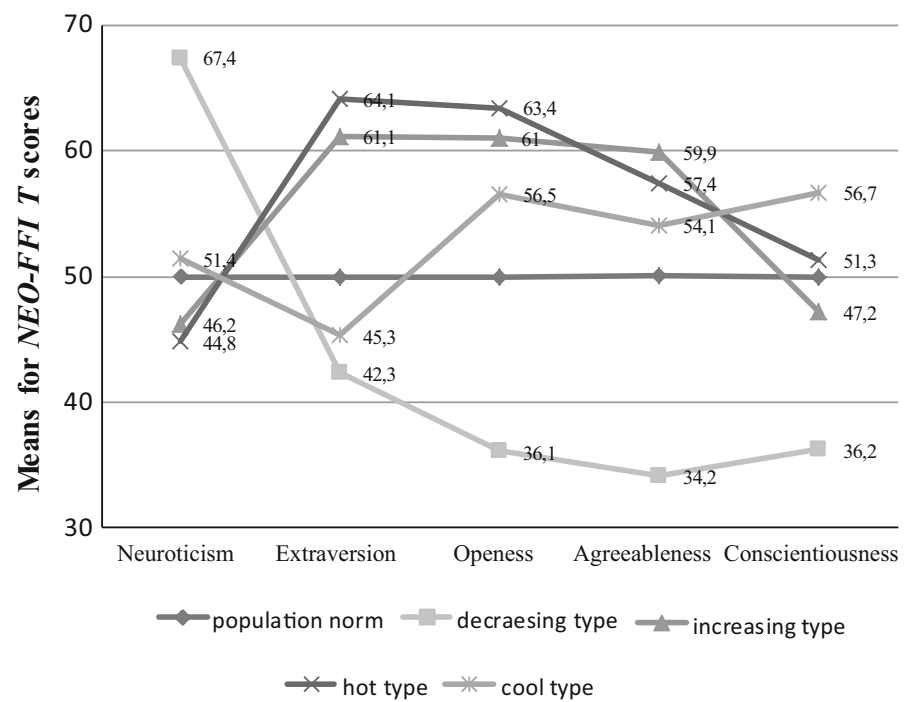

Fig. 3 General pattern of results obtained by individuals representing each cluster in NEO-FFI questionnaire 


\subsubsection{Patterns of Mood Regulation Tendencies and Mood Changes Measured Directly}

The four-ways ANOVAS for each mood dimension (HT, EA, TA) as dependent repeated variables [the level of mood after mood induction (1st measurement) and the level of mood after the task (2nd measurement), and regulative types (increasing, decreasing, hot and cool), type of mood induction (positive/negative) and the level of cognitive loading (high/ low cognitive loading condition) as independent variables] revealed significant effects of four way interactions for energetic arousal (EA) $\left[F_{(3,202)}=8.06 ; p<.01 ; \eta^{2}=.037\right]$, and tense arousal (TA) $\left[F_{(3,202)}=5.12 ; p<.01 ; \eta^{2}=.018\right]$. Post hoc analyses (see Fig. 4) pointed at essential differences between increasing and decreasing types, especially after positive mood induction $\left(F_{(2,98)}=7.24 ; p<.01 ; \eta^{2}=.023\right)$. While the level of EA decreased for the decreasing type in both high and low cognitive loading condition, it increased for the increasing type only in easy condition (see Fig. 4). Moreover, for the hot type energetic arousal increased in both low $(p<.05)$ and high $(p<.05)$ cognitive loading condition.
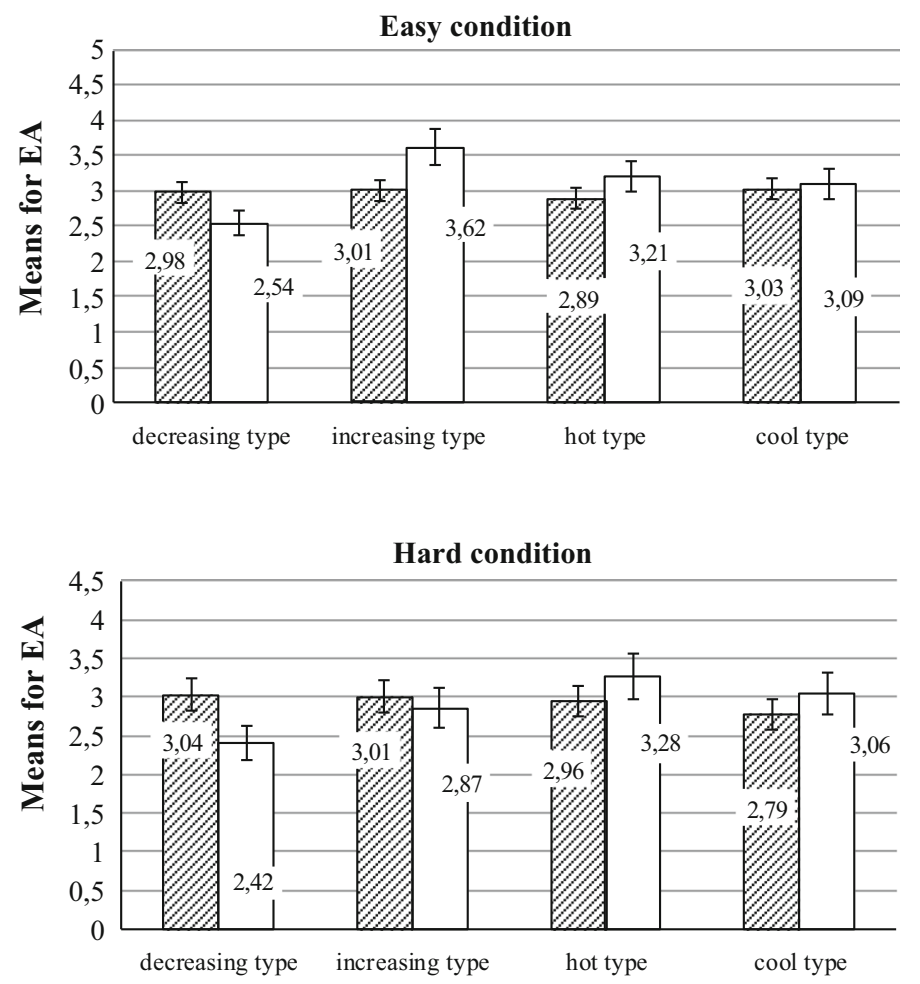

$\varpi$ measurement 1-MACL mood measurement after positive mood induction

$\square$ measurement 2 - MACL mood measurement after LDT

Fig. 4 Change of energetic arousal after positive mood induction (decreasing, increasing and hot types) 
The level of TA increased for the decreasing type in both high cognitive loading condition and low cognitive loading condition and it decreased for the increasing type only in easy condition (see Fig. 5).

\subsubsection{Patterns of Mood Regulation Tendencies and Mood Changes Measured Indirectly}

The 4 (regulative types) $\times 2$ (mood induction) $\times 2$ (the level of cognitive loading) mixed ANOVAs were performed on mean correct RTs data from nine stimuli blocks as dependent repeated variable independently for positive, negative and neutral stimuli.

In the analysis considering time reactions for negative stimuli (negative words) a significant effect of three-way interaction of moment of measure, mood induction and regulative types was revealed $\left[F_{(8,192)}=3.08 ; p<.05 ; \eta^{2}=.019\right]$. Generally, the decreasing type reacted faster for negative words than other groups $(p s<.05)$, but only in hard condition and after positive mood induction.
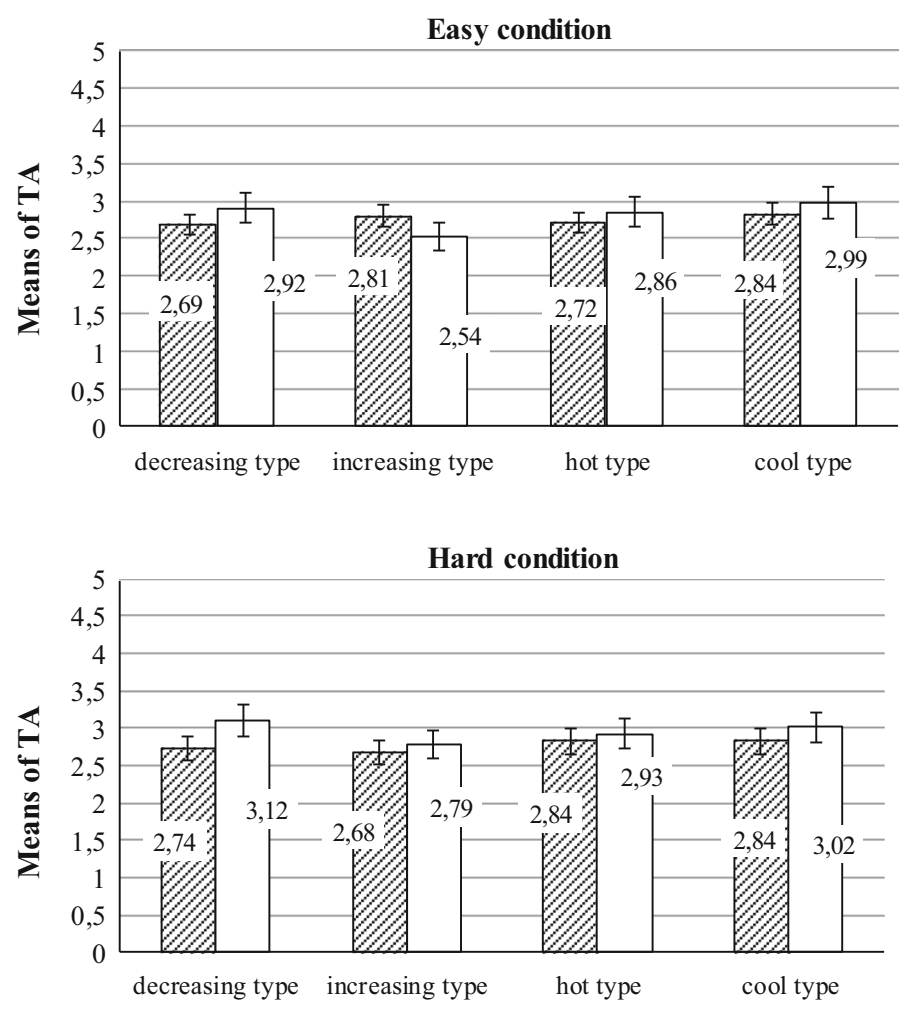

Q measurement 1- MACL mood measurement after positive mood induction

$\square$ measurement 2 - MACL mood measurement after LDT

Fig. 5 Change of tense arousal after positive mood induction (decreasing and increasing types) 
Tests of trends from the analysis of variance showed a highly significant linear trend for this three way interaction $\left[F_{(2,198)}=7.90 ; p<.001 ; \eta^{2}=.028\right]$. The analysis of simple effects showed that the decreasing type decreased time reaction for negative words after positive mood induction, in low $(p<.05)$ as well as in high $(p<.05)$ cognitive loading condition (see Fig. 6).

In the analysis considering time reactions for positive stimuli (positive words) significant effect of four-way interaction of the moment of measure, mood induction, the level of cognitive loading and regulative type was revealed $\left[F_{(8,192)}=2.57 ; p<.05 ; \eta=.024\right]$. The increasing type reacted generally faster for positive words than other groups ( $p s<.05)$, but only in easy condition and after positive mood induction.

A significant linear trend for this four-way interaction was revealed $\left[F_{(2,198)}=6,13\right.$; $\left.p<.01 ; \eta^{2}=.019\right]$. As the analysis of simple effects demonstrated, the increasing and hot

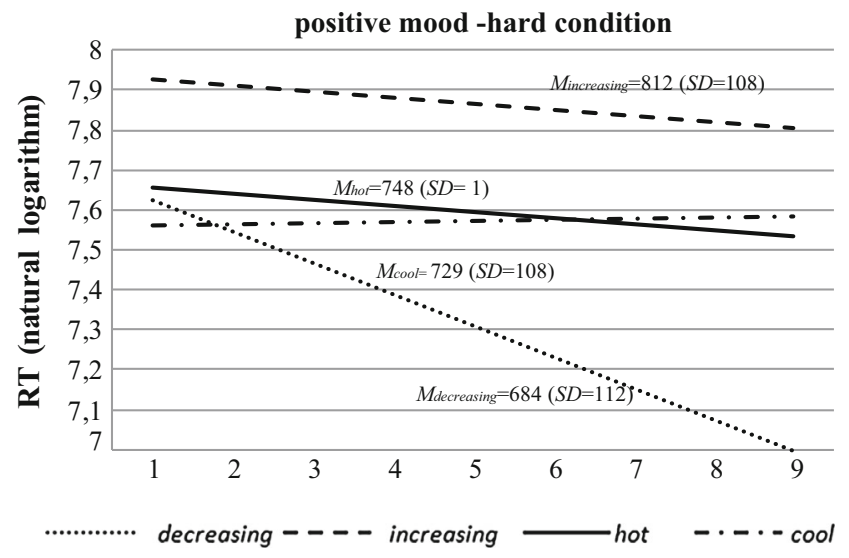

$M$ - mean reaction times for negative words in each cl uster

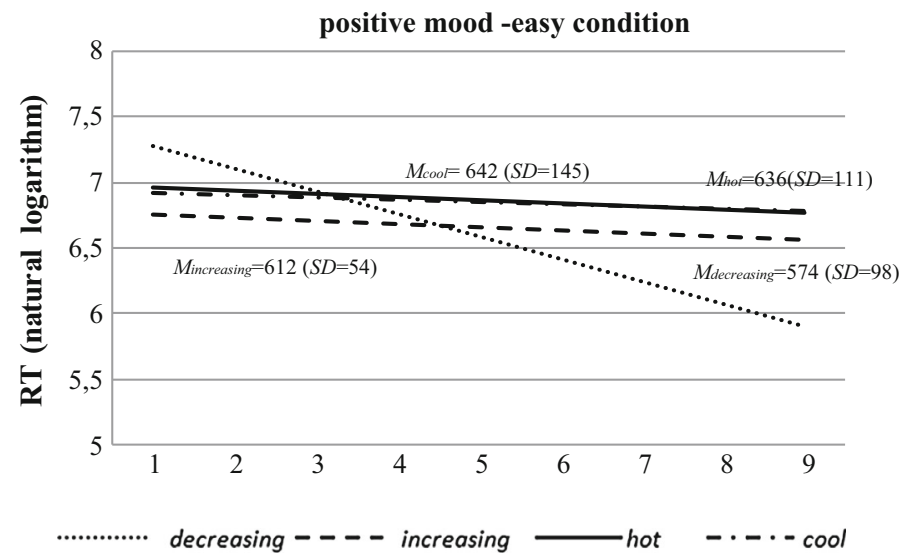

$M$ - mean reaction times for negative words in each cluster

Fig. 6 Linear trend for negative words after positive mood induction-low and high cognitive loading condition 
types decreased their RT's to positive words after positive mood induction but only in low cognitive loading condition ( $p s<.05$; see Fig. 7).

\subsubsection{Big Five Personality Traits, Patterns of Mood Regulation Tendencies and Mood Changes Measured Directly and Indirectly}

The five-way ANOVAs with the moment of direct or indirect mood measurement as a dependent repeated variable and regulative types (increasing, decreasing, hot and cool), type of mood induction (positive, negative), the level of cognitive loading (high and low cognitive loading condition) and the level of personality traits as independent variables were conducted.

The analysis considering direct mood measurement [each mood dimension (HT, EA, TA) as dependent repeated variable; the level of mood after mood induction (1st measurement) and the level of mood after the task (2nd measurement)] showed significant effects of the three way interaction of moment of measure, regulative types and personality traits for energetic arousal. We observed the following:

- A significant interaction of moment of measure, regulative types and Neuroticism, $F_{(2,202)}=3.08 ; p<.05 ; \eta^{2}=.016$. The analysis of simple effects showed that high level of neuroticism for the decreasing type promoted strong and statistically significant energetic arousal decrease $(p<.05)$. An analogical change of energetic arousal was not observed for low neurotic people representing the decreasing type $(p=.11)$. Neuroticism does not modify tense arousal changes-the level of this mood dimension decreased for the decreasing type independently of neuroticism level $(p>.05)$.

- An significant interaction of moment of measure, regulative types and Extraversion, $F_{(2,202)}=2.93 ; p<.05 ; \eta^{2}=.019$. For the decreasing type strong energetic arousal decrease was observed only for individuals low in extraversion $(p<.05)$, and not for those high in extraversion $(p=.09)$.

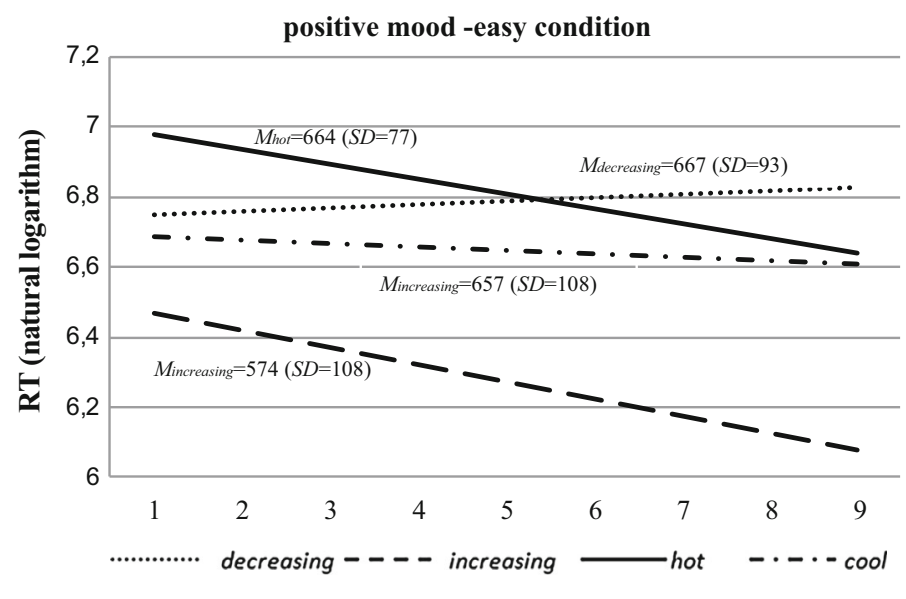

$M$ - mean reaction times for positive words in each cluster

Fig. 7 Linear trend for positive words after positive mood induction-low cognitive loading condition 
- A significant interaction of moment of measure, regulative types and Conscientiousness, $\mathrm{F}_{(2,202)}=2.87 ; p<.05 ; \eta^{2}=.021$. High level of this trait for the increasing type was related to the decrease of energetic arousal $(p<.05)$.

Analogical analyses for RTs for negative and positive words were conducted. No interactional effects of the analyzed independent variables on RTs for affective words were found (Figs. 8, 9, 10).

\section{Discussion}

The aims of the current study were to experimentally analyze the influence of different patterns of mood regulation tendencies, Big Five personality traits and their interaction on automatic and controlled mood changes. This is probably a pioneer study in the area of mood regulation where the criterion variable (mood changes) was measured (1) directly using self-report (MACL questionnaire), as well as (2) indirectly by means of emotional version of LDT.

Before referring to our specific hypotheses we would like to point at two important and more general results obtained in this study. Firstly, in the preliminary analysis we found that individual tendencies toward mood improvement/deterioration occur in four specific profiles labeled as increasing, decreasing, hot and cool. The increasing type is characterized by high tendency toward positive mood improvement and negative mood deterioration, whereas the decreasing type-by high tendency toward negative mood improvement and positive mood deterioration. Hot type individuals declare they often use strategies leading to mood improvement (positive mood improvement, negative mood deterioration) as well as mood deterioration (negative mood deterioration, positive mood improvement), whereas participants representing cool type use those two types of strategies very rarely. This research is the first to investigate these particular combinations of traits. Secondly, it seems that proposed procedure allowed us to grasp both automatic and

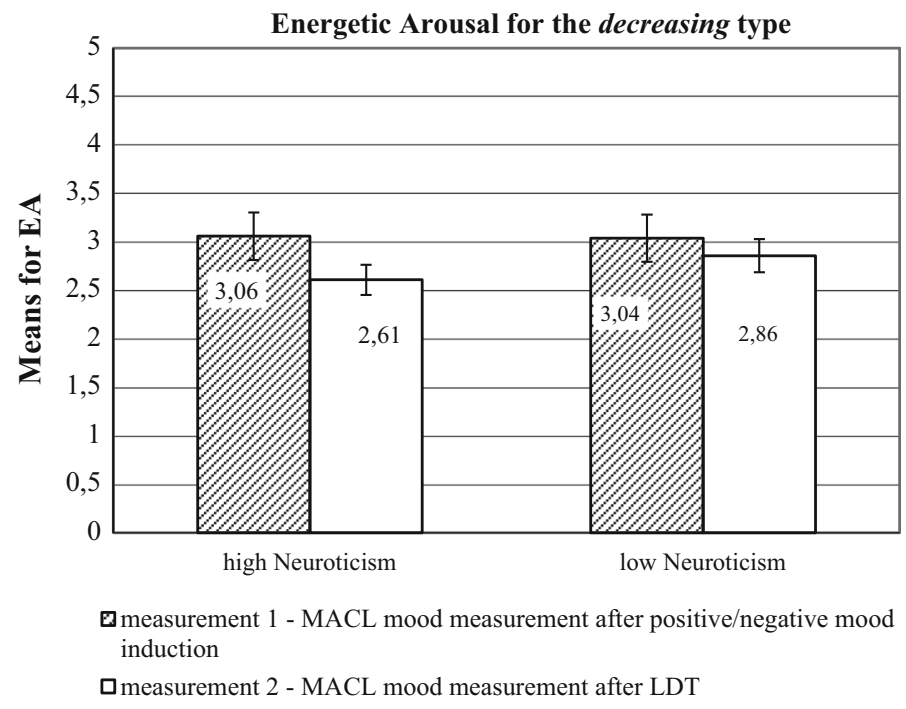

Fig. 8 Change of energetic arousal depending on the level of neuroticism for the decreasing type 


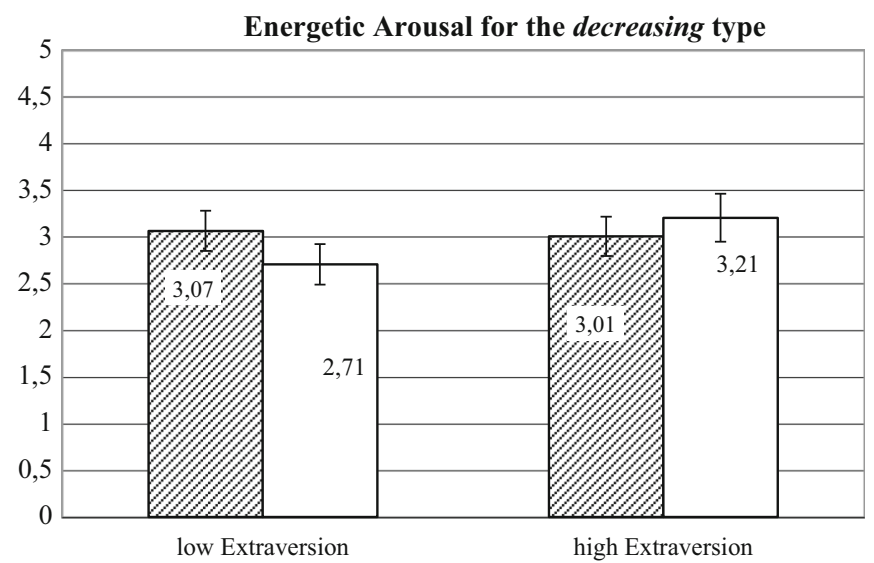

Đmeasurement 1 - MACL mood measurement after positive/negative mood induction

口measurement 2 - MACL mood measurement after LDT

Fig. 9 Change of energetic arousal depending on the level of extraversion for the decreasing and increasing type

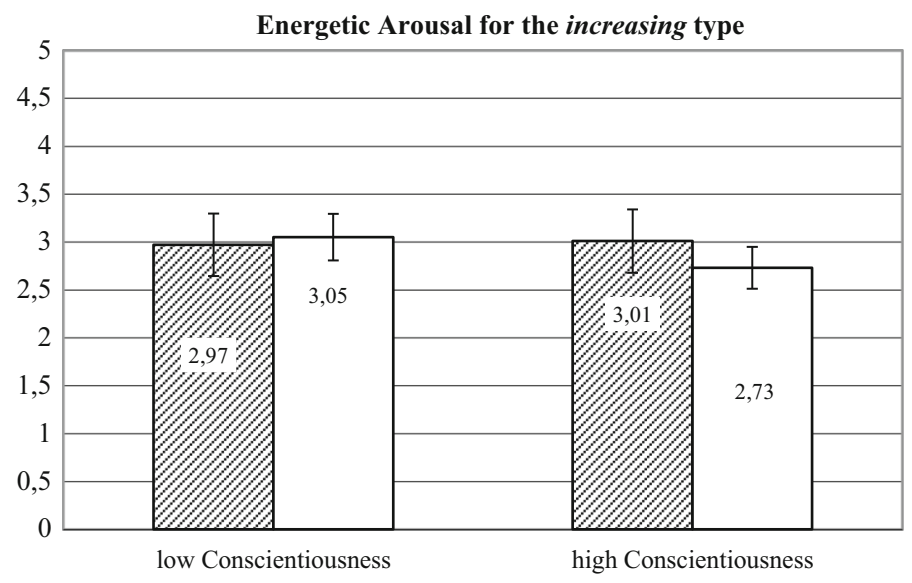

Đmeasurement 1 - MACL mood measurement after positive/negative mood induction

口measurement 2 - MACL mood measurement after LDT

Fig. 10 Change of energetic arousal depending on the level of conscientiousness for the increasing type

controlled mood regulation strategies. Mood changes observed especially for the decreasing type were registered in both high and low cognitive loading conditions. They manifested themselves in changes of the intensity of different mood dimensions as well as in changes of time reactions for adjectives in an emotional version of LDT. It revealed that the emotional version of LDT might be useful in examining the specificity of mood changes in full time dimension. It provides an opportunity to analyze temporal dynamics of 
mood regulation like time needed for recovery from induced emotions to optimal affective state without imposing self-insight (Lischetzke et al. 2011). More analyses are needed to assess validity and other possible applications of this mood measure in experimental studies.

Our results let us dispel some ambiguities concerning the impact of specific patterns of mood regulation tendencies on mood changes. The obtained data partially supported our predictions concerning mood regulation processes related to different mood regulation tendencies.

As we predicted in hypothesis 1 , mood changes related to high tendency toward mood improvement consisted in positive mood increasing - they lead to increased energetic arousal and decreased tense arousal only after positive mood induction and only in low cognitive loading condition. Thus, the specificity of positive mood changes characteristic for the increasing type involved psycho-physiological energy mobilization and tension reduction. Analogical mood changes indices for participants representing this type were obtained also on indirect mood measure-emotional version of the LDT. Positive mood improvement for the increasing type was expressed by the linear decrease of time reactions to positive target words. Unexpectedly, our analyses suggested that high mood improvement tendency in the increasing type was not related to negative mood deterioration processes after negative mood induction.

The obtained results partially supported hypothesis 2 . Mood changes observed for the decreasing people after positive mood induction and in both cognitive loading conditions manifested themselves in decreased energetic arousal and increased tense arousal. Thus the specificity of positive mood changes characteristic for this type involved especially physiological energy reduction and tension increase. Positive mood deterioration for the decreasing type was also expressed by the linear decrease of time reactions to negative target words during the LDT. Contrary to hypothesis 2 , we did not find any results pointing that high mood deterioration tendency in the decreasing type might lead to negative mood improvement processes. No specific patterns of time reactions were observed after negative mood induction for this type.

Taking into account the above results, we are able to draw some interesting conclusions. On the one hand, high mood deterioration tendency is not always definitely maladaptive, as it does not lead individuals to negative mood deterioration, whereas high mood improvement tendency is not always unequivocally positive, as it does not provoke negative mood repair. On the other hand, it may be possible that negative mood induced in this study was not sufficiently explicit or intensive to trigger mood regulation repair. Moreover, mood regulation processes directed toward negative mood increase are probably more strongly regulated by situational factors or/and characteristics of psychopathology (Larsen 2000). Such hypotheses should be explored more closely in future research.

The obtained data suggest that the concomitance of low levels of mood regulation tendencies is related with more stable mood experience. We did not find any statistically significant mood changes predicted in for the cool type (characterized by low level of tendency toward mood improvement as well as mood deterioration). Moreover, our study did not reveal any clear pattern of mood changes predicted in hypothesis 1 and 2 for the hot type (characterized by high level of tendency toward mood improvement as well as mood deterioration). Only one result suggests that individuals representing this cluster experienced mood improvement processes after positive mood induction-their level of energetic arousal increased in both high and low cognitive loading condition. It is possible that for individuals in the hot cluster mood changes diverge simultaneously in different 
directions or the specificity of mood changes depends more on situational factors. The consequence of being hot should be explored in future research.

Our study also explored relationships between Big Fiver personality traits and the specificity of mood changes. In hypothesis 3 we expected that mood changes for high neurotic persons would consist in positive mood deteriorating (after positive mood induction) and negative mood improving (after negative mood induction) whereas for high extraverts-in positive mood improving (after positive mood induction) and negative mood deteriorating (after negative mood induction). What emerged, however, was a little bit more complicated pattern of results. As predicted in hypothesis 3, high Neuroticism was conducive to a strong energetic arousal decrease after positive and negative mood induction. Surprisingly, this effect was observed only in participants representing the decreasing type. We did not find any results indicating that high level of Extraversion contributed to positive mood improving or/and negative mood deterioration. Conversely, our results suggest that introverts representing the decreasing type are much more vulnerable to strong energetic arousal decrease after positive as well as after negative mood induction. Additionally, we found that for the increasing type high level of Consciousness is related with energetic arousal decrease. We may assume that this personality trait "weakens" the adaptive meaning of high tendency toward mood improvement leading to the reduction of psycho-physiological energy.

In the present study we asked also about the specific impact of individual tendencies toward mood improvement/deterioration and Big Five personality traits on automatic and controlled mood changes (Q1). As our study showed, the status of mood changes (automatic versus controlled) is related especially to individual tendencies toward mood regulation. We found that mood improvement processes characteristic for the increasing type were observed only in low cognitive loading condition. Thus, they require the use of more motivated and more effortful but maybe not always strictly controlled mood management skills. We may suppose that mood deterioration processes-observed for the decreasing type in both cognitive loading conditions-are constituted especially from automatic mechanism starting up/maintaining cycle of negative affectivity. Future research should analyze more precisely why people representing the decreasing type have problems with "switching up" to mood improvement processes. It may be related with another important implication of our study. Results obtained from emotional version of LDT suggest that individual tendencies toward mood regulation are related to differences in reactivity to affective stimuli. The existing literature implicates different cognitive resources as a potential mechanism of mood regulation. Our experiment showed that mood regulation processes observed in different regulative types manifested themselves in changes of reaction times for words specifically related to optimal affective state (negative words for the decreasing type, positive words for the increasing type). It is possible that, as a result of greater experience engaging mood improvement action, those representing the increasing type have greater reactivity to positive stimuli and (likely) greater cognitive resources available for mood improvement. An analogical relation appears probably between the decreasing type and negative stimuli.

Our findings may delineate a new area of research concerning different contributors of well-being. As our results showed the objective of SWB is not always happiness (see also North et al. 2011). People may differ in stable tendencies toward mood regulation and these tendencies may promote different moods (positive or negative) as desired. In other words, different mood regulation tendencies seems to be related with well-being to the extent that they lead to some beneficial outcomes, which are not always related with 
positive mood and which are maintained and pursued with flexibility. This assumption needs further clarification and research.

The results presented here may have some practical implications. Many classical as well as modern theories of psychotherapy try to identify relations between non-adaptive patterns of affective regulation and psychopathology (e.g. Leahy et al. 2011). An essential part of treatment is helping individual to overcome non-adaptive schemes of mood regulation by eliminating strategies leading to mood deterioration (Leahy et al. 2011) or maintaining positive feeling (Tugade and Fredrickson 2004). Our findings suggest that therapeutic techniques should concentrate on a wide variety of mood regulation strategies (not only related with mood improvement) and matched to the individual characteristic of the patient.

Although the results of the present study are promising, in the following we will discuss some limitations. One may be related with mood regulation tendencies measurement. To put it simply, are people always consciously aware of using strategies to influence their own mood? It is possible that some of the strategies assessed in the MRS scale, particularly those more popular, are used relatively habitually (Gross 1998) and people may not be explicitly aware that they are using such behaviors in order to regulate affect? Moreover, our conclusions might be limited by the procedure complexity which could interfere with the process of automatic as well as controlled mood regulation. Thus, we cannot explicitly conclude whether all observed mood changes were due to mood regulation processes or procedural factors.

Acknowledgements The research was a part of Project "Mood: their regulative and informative functions" supported by the Committee of Scientific Research in Poland (Grant No. 1410/B/H03/2009/37), and accepted by the Committee of Research Ethics of SWPS University.

Open Access This article is distributed under the terms of the Creative Commons Attribution 4.0 International License (http://creativecommons.org/licenses/by/4.0/), which permits unrestricted use, distribution, and reproduction in any medium, provided you give appropriate credit to the original author(s) and the source, provide a link to the Creative Commons license, and indicate if changes were made.

\section{References}

Arhaud-Day, M. L., Rode, J. C., Mooney, C. H., \& Hear, J. P. (2005). The subjective wellbeing construct: A test of its convergent, discriminant, and factorial validity. Social Indicators Research, 74, 445-476.

Augustine, A. A., \& Larsen, R. J. (2015). Personality, affect, and affect regulation. In M. Mikulincer, P. R. Shaver, M. L. Cooper, \& R. J. Larsen (Eds.), APA handbook of personality and social psychology, Volume 4: Personality processes and individual differences (pp. 147-165). Washington, DC: American Psychological Association.

Coifman, K. G., Bonanno, G. A., Ray, R. D., \& Gross, J. J. (2007). Does repressive coping promote resilience? Affective - automatic response discrepancy during bereavement. Journal of Personality and Social Psychology, 92, 745-758.

Costa, P. T., Jr., \& McCrae, R. R. (1992). Revised NEO personality inventory (NEO-PI-R) and NEO fivefactor inventory (NEO-FFI) professional manual. Odessa, FL: Psychological Assessment Resources.

Diener, E. (1999). Introduction to the special section on the structure of emotions. Journal of Personality and Social Psychology, 76, 603-804.

Feldman-Barrett, L. F., Mesquita, B., Ochsner, K. N., \& Gross, J. J. (2007). The experience of emotion. Annual Review of Psychology, 58, 373-403.

Garnefski, N., Kraaij, V., \& Spinhoven, Ph. (2001). Negative life events, cognitive emotion regulation and emotional problems. Personality and Individual Differences, 30, 1311-1327.

Gohm, C. L. (2003). Mood regulation and emotional intelligence: Individual differences. Journal of Personality and Social Psychology, 84, 594-607. 
Gorynska, E. (2005). UWIST Mood Adjective Check List. Manual. Warsaw: Psychological Test Laboratory of PPA.

Gross, J. J. (1998). The emerging field of emotion regulation: An integrative review. Review of General Psychology, 2, 271-299.

Gross, J. J., \& John, O. P. (2003). Individual differences in two emotion regulation processes: Implications for affect, relationships, and well-being. Journal of Personality and Social Psychology, 85, 348-362.

Gyurak, A., Gross, J. J., \& Etkin, A. (2011). Explicit and implicit emotion regulation: A dual-process framework. Cognition and Emotion, 25(400-412), 10.

Haga, S. M., Kraft, P., \& Corby, K. (2009). Emotion regulation: Antecedents and well-being outcomes of cognitive reappraisal and expressive supression in cross-cultural samples. Journal of Happiness Studies, 10, 271-291.

Halberstadt, J. B., Niedenthal, P. M., \& Kushner, J. (1995). Resolution of lexical ambiguity by emotional state. American Psychological Society, 6, 278-282.

Isen, A. M. (1987). Positive affect, cognitive processes, and social behavior. In L. Berkowitz (Ed.), Advances in experimental social psychology (pp. 203-253). San Diego, CA: Academic Press.

John, O. P., \& Gross, J. J. (2007). Individual differences in emotion regulation: Links to global trait, dynamic, and social cognitive constructs. In J. J. Gross (Ed.), Handbook of emotion regulation (pp. 351-372). New York: Guilford.

Joormann, J. (2006). The relation of rumination and inhibition: Evidence from a negative priming task. Cognitive Therapy and Research, 30, 149-160.

Kashdan, T. B. (2007). New developments in emotion regulation with emphasis on the positive spectrum of human functioning. Journal of Happiness Studies, 8, 303-310.

Larsen, R. J. (2000). Toward a science of mood regulation. Psychological Inquiry, 11(3), 129-141.

Leahy, R., Tirch, D., \& Napolitano, A. (2011). Emotion regulation in psychotherapy. A practitioner's guide. The Guilford Press: New York.

Lischetzke, T., Angelova, R., \& Eid, M. (2011). Validating an indirect measure of clarity of feelings: Evidence from laboratory and naturalistic settings. Psychological Assessment, 23(2), 447-455.

Matthews, G., Deary, I. J., \& Whiteman, M. C. (2003). Personality traits (2nd ed.). Cambridge: Cambridge University Press.

Matthews, G., Jones, D. M., \& Chamberlain, A. G. (1990). Refining the measurement of mood: The UWIST mood adjective checklist. British Journal of Psychology, 81, 17-42.

Mauss, I. B., Bunge, S. A., \& Gross, J. J. (2007). Automatic emotion regulation. Social and Personality Psychology Compass, 1, 1-22.

Niedenthal, P. M., \& Setterlund, M. B. (1994). Emotion congruence in perception. Personality and Social Psychology Bulletin, 20, 401-411.

North, R. J., Pai, A. V., Hixon, J., \& Holahan, C. J. (2011). Finding happiness in negative emotions: An experimental test of a novel expressive writing paradigm. Journal of Positive Psychology, 6(3), $192-203$.

Nowicka, M. (2009). Mood regulation practices questionnaire. Construction and psychometric validity. Psychological Studies, 47(3), 61-69.

Panksepp, J. (1993). Neurochemical control of moods and emotions: Amino acids to neuropeptides. In M. Lewis \& J. Haviland (Eds.), The handbook of emotions (pp. 87-107). New York: Guilford Press.

Russell, J. A. (2003). Core affect and the psychological construction of emotion. Psychological Review, 110, $145-172$.

Rusting, C. L., \& De Hart, T. (2000). Retrieving positive memories to regulate negative mood: Consequences for mood-congruent memory. Journal of Personality and Social Psychology, 78, 737-752.

Rusting, C., \& Larsen, R. J. (1995). Moods as sources of stimulation: Relationships between personality and desired mood states. Personality and Individual Differences, 18, 321-329.

Salovey, P., Mayer, J. D., Goldman, S., Turvey, C., \& Palfai, T. (1995). Emotional attention, clarity and repair: Exploring emotional intelligence using the trait meta-mood scale. In J. D. Pennebaker (Ed.), Emotion, disclosure, and health (pp. 125-154). Washington: American Psychological Association.

Scheier, M. F., Carver, C. S., \& Bridges, M. (1994). Distinguishing optimism from neuroticism (and trait anxiety, self-mastery, and self-esteem): A reevaluation of the life orientation test. Journal of Personality and Social Psychology, 67, 1063-1078.

Schimmack, U., \& Grob, A. (2000). Dimensional models of core affect: A quantitative comparison by means of structural equation modeling. European Journal of Personality, 14, 325-345.

Thayer, R. E. (1989). The biopsychology of mood and activation. New York: Oxford University Press.

Tobin, R., Graziano, W., Vanman, E., \& Tassinary, L. G. (2000). Personality, emotional experience, and efforts to control emotions. Journal of Personality and Social Psychology, 79, 656-669. 
Tomyn, A. J., \& Cummins, R. (2011). Subjective wellbeing and homeostatically protected mood: Theory validation with adolescents. Journal of Happiness Studies, 12(5), 897-914.

Tugade, M. M., \& Fredrickson, B. L. (2004). Resilient individuals use positive emotions to bounce back from negative emotional experiences. Journal of Personality and Social Psychology, 86, 320-333.

Watson, D., \& Clark, L. A. (1997). Extraversion and its positive emotional core. In R. Hogan, J. Johnson, \& S. Briggs (Eds.), Handbook of personality psychology (pp. 767-793). San Diego, CA: Academic Press.

Watson, D., \& Tellegen, A. (1985). Toward a consensual structure of mood. Psychological Bulletin, 98, 219-235.

Watson, D., Wiese, D., Vaidya, J., \& Tellegen, A. (1999). The two general activation systems of affect: Structural findings, evolutionary considerations and psychobiological evidence. Journal of Personality and Social Psychology, 76, 820-838.

Wojciszke, B. (2003). Mood regulation scales. In M. Marszal-Wisniewska, T. Klonowicz, \& M. FajkowskaStanik (Eds.), Psychology of individual differences (pp. 163-178). Gdansk: GWP.

Yik, M., \& Russell, J. A. (2001). Predicting the big two of affect from the big five of personality. Journal of Research in Personality, 35, 247-277.

Yik, M., Steiger, J. H., \& Russell, J. A. (2011). A 12-point circumflex structure of core affect. Emotion, 11(4), 705-731.

Zajenkowski, M., Gorynska, E., \& Winiewski, M. (2012). Variability of the relationship between personality and mood. Personality and Individual Differences, 52, 858-861.

Zawadzki, B., Szczepaniak, P., \& Strelau, J. (1995). Psychological diagnosis of Big Five factors of personality: Polish adaptation of NEO-FFI questionnaire of Costa and McCrae. Psychological Studies, 33, 189-225. 